\title{
Fuzzy Differential Subordinations Results for $\lambda$-pseudo Starlike and $\lambda$-pseudo Convex Functions with Respect to Symmetrical Points
}

\author{
Abbas Kareem Wanas ${ }^{1}$ and Dhirgam Allawy Hussein ${ }^{2}$ \\ ${ }^{1}$ Department of Mathematics, College of Science, University of Al-Qadisiyah, Iraq \\ e-mail: abbas.kareem.w@qu.edu.iq \\ ${ }^{2}$ Directorate of Education in Al-Qadisiyah, Diwaniyah, Iraq \\ e-mail: dhirgam82@gmail.com
}

\section{Abstract}

In the present work, we establish some fuzzy differential subordination results for $\lambda$-pseudo starlike and $\lambda$-pseudo convex functions with respect to symmetrical points in the open unit disk.

\section{Introduction and Preliminaries}

Let $T$ indicate the family of functions $f$ and has the series form:

$$
f(z)=z+\sum_{n=2}^{\infty} a_{n} z^{n},
$$

which are analytic and univalent in the open unit disk $U=\{z \in \mathbb{C}:|z|<1\}$.

For functions $f_{j} \in T(j=1,2)$ given by

$$
f_{j}(z)=z+\sum_{n=2}^{\infty} a_{n, j} z^{n} \quad(j=1,2),
$$

Received: March 15, 2020; Accepted: April 8, 2020

2010 Mathematics Subject Classification: 30C45.

Keywords and phrases: fuzzy set, fuzzy differential subordination, $\lambda$-pseudo starlike with respect to symmetrical points, $\lambda$-pseudo convex with respect to symmetrical points, fuzzy best dominant.

Copyright (C) 2020 Abbas Kareem Wanas and Dhirgam Allawy Hussein. This is an open access article distributed under the Creative Commons Attribution License, which permits unrestricted use, distribution, and reproduction in any medium, provided the original work is properly cited. 
the Hadamard product of $f_{1}$ and $f_{2}$ is defined by

$$
\left(f_{1} * f_{2}\right)(z)=z+\sum_{n=2}^{\infty} a_{n, 1} a_{n, 2} z^{n}=\left(f_{2} * f_{1}\right)(z) .
$$

A function $f \in T$ is called starlike with respect to symmetrical points, if (see [8])

$$
\operatorname{Re}\left\{\frac{z f^{\prime}(z)}{f(z)-f(-z)}\right\}>0, \quad z \in U
$$

The family of all such functions is denote by $S_{S}^{*}$.

The family of starlike functions with respect to symmetrical points obviously includes the family of convex functions with respect to symmetrical points, $C_{S}$, satisfying the following condition:

$$
\operatorname{Re}\left\{\frac{\left(z f^{\prime}(z)\right)^{\prime}}{(f(z)-f(-z))}\right\}>0, \quad z \in U .
$$

Recently, Babalola [1] defined the class $\mathcal{L}_{\lambda}$ of $\lambda$-pseudo-starlike functions as follows:

Let $f \in T$ and $\lambda \geq 1$. Then $f \in \mathcal{L}_{\lambda}$ of $\lambda$-pseudo-starlike functions in $U$ if and only if

$$
\operatorname{Re}\left\{\frac{z\left(f^{\prime}(z)\right)^{\lambda}}{f(z)}\right\}>0, \quad z \in U
$$

Definition 1.1 [9]. Let $X$ be a non-empty set. An application $F: X \rightarrow[0,1]$ is called fuzzy subset. An alternate definition, more precise, would be the following:

A pair $\left(A, F_{A}\right)$, where $F_{A}: X \rightarrow[0,1]$ and $A=\left\{x \in X: 0<F_{A}(x) \leq 1\right\}=$ $\operatorname{supp}\left(A, F_{A}\right)$ is called fuzzy subset. The function $F_{A}$ is called membership function of the fuzzy subset $\left(A, F_{A}\right)$.

Definition 1.2 [5]. Let two fuzzy subsets of $X,\left(M, F_{M}\right)$ and $\left(N, F_{N}\right)$. We say that the fuzzy subsets $M$ and $N$ are equal if and only if $F_{M}(x)=F_{N}(x), x \in X$ and we denote this by $\left(M, F_{M}\right)=\left(N, F_{N}\right)$. The fuzzy subset $\left(M, F_{M}\right)$ is contained in the 
fuzzy subset $\left(N, F_{N}\right)$ if and only if $F_{M}(x) \leq F_{N}(x), x \in X$ and we denote the inclusion relation by $\left(M, F_{M}\right) \subseteq\left(N, F_{N}\right)$.

Let $D \subseteq \mathbb{C}$ and $f, g$ analytic functions. We denote by

$$
f(D)=\operatorname{supp}\left(f(D), F_{f(D)}\right)=\left\{f(z): 0<F_{f(D)}(f(z)) \leq 1, z \in D\right\}
$$

and

$$
g(D)=\operatorname{supp}\left(g(D), F_{g(D)}\right)=\left\{g(z): 0<F_{g(D)}(g(z)) \leq 1, z \in D\right\} .
$$

Definition 1.3 [5]. Let $D \subseteq \mathbb{C}, z_{0} \in D$ be a fixed point, and let the functions $f, g \in \mathcal{H}(D)$. The function $f$ is said to be fuzzy subordinate to $g$ and write $f \prec_{F} g$ or $f(z) \prec_{F} g(z)$ if the following conditions are satisfied:

1. $f\left(z_{0}\right)=g\left(z_{0}\right)$,

2. $F_{f(D)}(f(z)) \leq F_{g(D)}(g(z)), z \in D$.

Definition 1.4 [6]. Let $\psi: \mathbb{C}^{3} \times U \rightarrow \mathbb{C}$ and let $h$ be univalent in $U$. If $p$ is analytic in $U$ and satisfies the (second-order) fuzzy differential subordination:

$$
F_{\psi\left(\mathbb{C}^{3} \times U\right)}\left(\psi\left(p(z), z p^{\prime}(z), z^{2} p^{\prime \prime}(z) ; z\right)\right) \leq F_{h(U)}(h(z)),
$$

i.e.,

$$
\psi\left(p(z), z p^{\prime}(z), z^{2} p^{\prime \prime}(z) ; z\right) \prec_{F} h(z), \quad z \in U,
$$

then $p$ is called a fuzzy solution of the fuzzy differential subordination. The univalent function is $q$ called a fuzzy dominant of the fuzzy solutions of the fuzzy differential subordination, or more simple a fuzzy dominant, if $p(z) \prec_{F} q(z), z \in U$ for all $p$ satisfying (1.2). A fuzzy dominant $\tilde{q}$ that satisfies $\tilde{q}(z) \prec_{F} q(z), z \in U$ for all fuzzy dominant $q$ of (1.2) is said to be the fuzzy best dominant of (1.2).

Lemma 1.1 [2]. Let $q$ be univalent in $U$ and let $\theta$ and $\phi$ be analytic in a domain $D$ containing $q(U)$ with $\phi(w) \neq 0$ when $w \in q(U)$. Set $Q(z)=z q^{\prime}(z) \phi(q(z))$ and $h(z)=\theta(q(z))+Q(z)$. Suppose that

(1) $Q(z)$ is starlike in $U$, 
(2) $\operatorname{Re}\left\{\frac{z h^{\prime}(z)}{Q(z)}\right\}>0$ for $z \in U$.

If $p$ is analytic in $U$, with $p(0)=q(0), p(U) \subset D$ and $\psi: \mathbb{C}^{2} \times U \rightarrow \mathbb{C}$, $\psi\left(p(z), z p^{\prime}(z)\right)=\theta(p(z))+z p^{\prime}(z) \cdot \phi(p(z))$ is analytic in $U$, then

$$
F_{\psi\left(\mathbb{C}^{2} \times U\right)}\left[\theta(p(z))+z p^{\prime}(z) \cdot \phi(p(z))\right] \leq F_{h(U)} h(z),
$$

implies

$$
F_{p(U)} p(z) \leq F_{q(U)} q(z),
$$

i.e., $p(z) \prec_{F} q(z)$ and $q$ is the fuzzy best dominant, where

$$
\begin{aligned}
\psi\left(\mathbb{C}^{2} \times U\right) & =\operatorname{supp}\left(\mathbb{C}^{2} \times U, F_{\psi\left(\mathbb{C}^{2} \times U\right)} \psi\left(p(z), z p^{\prime}(z)\right)\right) \\
& =\left\{z \in \mathbb{C}: 0<F_{\psi\left(\mathbb{C}^{2} \times U\right)} \psi\left(p(z), z p^{\prime}(z)\right) \leq 1\right\},
\end{aligned}
$$

and

$$
h(U)=\operatorname{supp}\left(U, F_{h(U)} h(z)\right)=\left\{z \in \mathbb{C}: 0<F_{h(U)} h(z) \leq 1\right\} .
$$

Recently, Oros and Oros [6, 7], Lupaş [3, 4] and Wanas and Majeed [10, 11] have obtained fuzzy differential subordination results for certain classes of analytic functions.

\section{Main Results}

Theorem 2.1. Suppose that $\alpha, \beta, \gamma \in \mathbb{C}, \delta>0, \lambda \geq 1, t \in \mathbb{C} \backslash\{0\}$ and $q$ be univalent function in $U$ such that $q(0)=1, q(z) \neq 0$ and

$$
\operatorname{Re}\left\{1+\frac{\beta}{t}(\gamma-1)+\frac{\alpha \gamma}{t} q(z)+(\gamma-2) \frac{z q^{\prime}(z)}{q(z)}+\frac{z q^{\prime \prime}(z)}{q^{\prime}(z)}\right\}>0 .
$$

Assume that $z(q(z))^{\gamma-2} q^{\prime}(z)$ is starlike in $U$. If $f \in T$ and $\Phi(\alpha, \beta, \gamma, \lambda, \delta, t ; z)$ is analytic in $U$, where

$$
\Phi(\alpha, \beta, \gamma, \lambda, \delta, t ; z)=\left(\frac{2 z\left(f^{\prime}(z)\right)^{\lambda}}{f(z)-f(-z)}\right)^{\gamma \delta}\left[\alpha+\beta\left(\frac{f(z)-f(-z)}{2 z\left(f^{\prime}(z)\right)^{\lambda}}\right)^{\delta}\right.
$$




$$
\begin{aligned}
& +t \delta\left(\frac{f(z)-f(-z)}{2 z\left(f^{\prime}(z)\right)^{\lambda}}\right)^{\delta} \\
& \left.\times\left(1+\frac{\lambda z f^{\prime \prime}(z)}{f^{\prime}(z)}-\frac{2(f(z)-f(-z))^{\prime}}{f(z)-f(-z)}\right)\right],
\end{aligned}
$$

then

$$
\begin{aligned}
F_{\psi\left(\mathbb{C}^{2} \times U\right)}[\Phi(\alpha, \beta, \gamma, \lambda, \delta, t ; z)] & \leq F_{\psi\left(\mathbb{C}^{2} \times U\right)}\left[(q(z))^{\gamma}\left(\alpha+\frac{\beta}{q(z)}+t \frac{z q^{\prime}(z)}{(q(z))^{2}}\right)\right] \\
& =F_{h(U)} h(z)
\end{aligned}
$$

implies

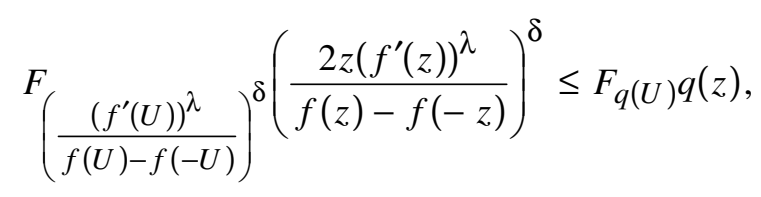

i.e.,

$$
\left(\frac{2 z\left(f^{\prime}(z)\right)^{\lambda}}{f(z)-f(-z)}\right)^{\delta} \prec_{F} q(z), \quad(z \in U)
$$

and $q$ is the fuzzy best dominant.

Proof. For given $f \in T$, define $p$ by

$$
p(z)=\left(\frac{2 z\left(f^{\prime}(z)\right)^{\lambda}}{f(z)-f(-z)}\right)^{\delta}
$$

It is clear that the function $p$ is analytic in $U$ and $p(0)=1$. Simple calculations show that

$$
(p(z))^{\gamma}\left(\alpha+\frac{\beta}{p(z)}+t \frac{z p^{\prime}(z)}{(p(z))^{2}}\right)=\Phi(\alpha, \beta, \gamma, \lambda, \delta, t ; z)
$$

where $\Phi(\alpha, \beta, \gamma, \lambda, \delta, t ; z)$ is given by (2.2). 
In the light of (2.3) and (2.5), we conclude that

$$
\begin{aligned}
& F_{\psi\left(\mathbb{C}^{2} \times U\right)}\left[(p(z))^{\gamma}\left(\alpha+\frac{\beta}{p(z)}+t \frac{z p^{\prime}(z)}{(p(z))^{2}}\right)\right] \\
\leq & F_{\psi\left(\mathbb{C}^{2} \times U\right)}\left[(q(z))^{\gamma}\left(\alpha+\frac{\beta}{q(z)}+t \frac{z q^{\prime}(z)}{(q(z))^{2}}\right)\right] .
\end{aligned}
$$

Define the functions $\theta$ and $\phi$ by

$$
\theta(w)=(\alpha w+\beta) w^{\gamma-1} \text { and } \phi(w)=t w^{\gamma-2} \text {. }
$$

Evidently, the functions $\theta$ and $\phi$ are analytic in $D=\mathbb{C} \backslash\{0\}$ and $\phi(w) \neq 0, w \in D$. Also, we find that

$$
Q(z)=z q^{\prime}(z) \phi(q(z))=t z(q(z))^{\gamma-2} q^{\prime}(z)
$$

and

$$
h(z)=\theta(q(z))+Q(z)=(q(z))^{\gamma}\left(\alpha+\frac{\beta}{q(z)}+t \frac{z q^{\prime}(z)}{(q(z))^{2}}\right) .
$$

Since $z(q(z))^{\gamma-2} q^{\prime}(z)$ is starlike univalent in $U$, we observe that $Q$ is starlike univalent in $U$.

$$
\operatorname{Re}\left\{\frac{z h^{\prime}(z)}{Q(z)}\right\}=\operatorname{Re}\left\{1+\frac{\beta}{t}(\gamma-1)+\frac{\alpha \gamma}{t} q(z)+(\gamma-2) \frac{z q^{\prime}(z)}{q(z)}+\frac{z q^{\prime \prime}(z)}{q^{\prime}(z)}\right\} .
$$

Now from (2.1) and (2.6), it is evident that

$$
\operatorname{Re}\left\{\frac{z h^{\prime}(z)}{Q(z)}\right\}>0 .
$$

On the application of Lemma 1.1, yields $F_{p(U)} p(z) \leq F_{q(U)} q(z)$. By using (2.4), we obtain

$$
F_{\left(\frac{\left(f^{\prime}(U)\right)^{\lambda}}{f(U)-f(-U)}\right)^{\delta}\left(\frac{2 z\left(f^{\prime}(z)\right)^{\lambda}}{f(z)-f(-z)}\right)^{\delta} \leq F_{q(U)} q(z)}
$$


i.e. $\left(\frac{2 z\left(f^{\prime}(z)\right)^{\lambda}}{f(z)-f(-z)}\right)^{\delta} \prec_{F} q(z)$ and $q$ is the fuzzy best dominant.

By putting the fuzzy dominant $q(z)=\frac{1+z}{1-z}, \gamma=t=1$ and $\alpha=\beta=0$ in Theorem 2.1, we obtain the following results:

Corollary 2.1. Let $\operatorname{Re}\left\{\frac{1+z^{2}}{1-z^{2}}\right\}>0$. If $f \in T$ and

$$
\delta\left(1+\frac{\lambda z f^{\prime \prime}(z)}{f^{\prime}(z)}-\frac{z(f(z)-f(-z))^{\prime}}{f(z)-f(-z)}\right)
$$

is analytic in $U$, then

$$
F_{\psi\left(\mathbb{C}^{2} \times U\right)}\left[\delta\left(1+\frac{\lambda z f^{\prime \prime}(z)}{f^{\prime}(z)}-\frac{z(f(z)-f(-z))^{\prime}}{f(z)-f(-z)}\right)\right] \leq F_{\psi\left(\mathbb{C}^{2} \times U\right)}\left[\frac{2 z}{1-z^{2}}\right],
$$

implies

$$
\left(\frac{2 z\left(f^{\prime}(z)\right)^{\lambda}}{f(z)-f(-z)}\right)^{\delta} \prec_{F} \frac{1+z}{1-z}, \quad(z \in U)
$$

and $q(z)=\frac{1+z}{1-z}$ is the fuzzy best dominant.

Theorem 2.2. Suppose that $\alpha, \beta, \gamma \in \mathbb{C}, \delta>0, \lambda \geq 1, t \in \mathbb{C} \backslash\{0\}$ and $q$ is univalent function in $U$ such that $q(0)=1, q(z) \neq 0$ and let $q$ satisfy (2.1). Assume that $z(q(z))^{\gamma-2} q^{\prime}(z)$ is starlike in $U$. If $f \in T$ and $\Psi(\alpha, \beta, \gamma, \lambda, \delta, t ; z)$ is analytic in $U$, where

$$
\begin{aligned}
\Psi(\alpha, \beta, \gamma, \lambda, \delta, t ; z)= & \left(\frac{2\left(\left(z f^{\prime}(z)\right)^{\prime}\right)^{\lambda}}{(f(z)-f(-z))^{\prime}}\right)^{\gamma \delta}\left[\alpha+\beta\left(\frac{(f(z)-f(-z))^{\prime}}{2\left(\left(z f^{\prime}(z)\right)^{\prime}\right)^{\lambda}}\right)^{\delta}\right. \\
& +t \delta\left(\frac{(f(z)-f(-z))^{\prime}}{2\left(\left(z f^{\prime}(z)\right)^{\prime}\right)^{\lambda}}\right)^{\delta}
\end{aligned}
$$




$$
\left.\times\left(\frac{\lambda z\left(z f^{\prime \prime \prime}(z)+2 f^{\prime}(z)\right)}{z f^{\prime \prime}(z)+f^{\prime}(z)}-\frac{z(f(z)-f(-z))^{\prime \prime}}{(f(z)-f(-z))^{\prime}}\right)\right]
$$

then

$$
\begin{aligned}
F_{\psi\left(\mathbb{C}^{2} \times U\right)}[\Psi(\alpha, \beta, \gamma, \lambda, \delta, t ; z)] & \leq F_{\psi\left(\mathbb{C}^{2} \times U\right)}\left[(q(z))^{\gamma}\left(\alpha+\frac{\beta}{q(z)}+t \frac{z q^{\prime}(z)}{(q(z))^{2}}\right)\right] \\
& =F_{h(U)} h(z),
\end{aligned}
$$

implies

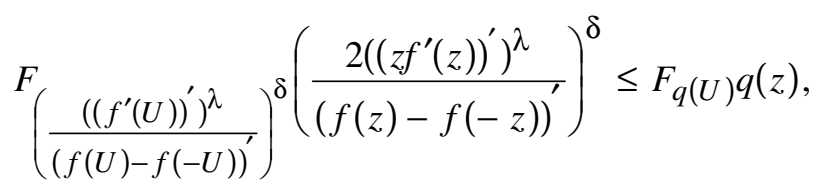

i.e.,

$$
\left(\frac{2\left(\left(z f^{\prime}(z)\right)^{\prime}\right)^{\lambda}}{(f(z)-f(-z))^{\prime}}\right)^{\delta} \prec_{F} q(z), \quad(z \in U)
$$

and $q$ is the fuzzy best dominant.

Proof. For given $f \in T$, define $p$ by

$$
p(z)=\left(\frac{2\left(\left(z f^{\prime}(z)\right)^{\prime}\right)^{\lambda}}{(f(z)-f(-z))^{\prime}}\right)^{\delta}
$$

It is obvious that the function $p$ is analytic in $U$ and $p(0)=1$. After some computations, we deduce that

$$
(p(z))^{\gamma}\left(\alpha+\frac{\beta}{p(z)}+t \frac{z p^{\prime}(z)}{(p(z))^{2}}\right)=\Psi(\alpha, \beta, \gamma, \lambda, \delta, t ; z),
$$

where $\Psi(\alpha, \beta, \gamma, \lambda, \delta, t ; z)$ is given by (2.7).

By making use of (2.8) and (2.10), it follows that

$$
F_{\psi\left(\mathbb{C}^{2} \times U\right)}\left[(p(z))^{\gamma}\left(\alpha+\frac{\beta}{p(z)}+t \frac{z p^{\prime}(z)}{(p(z))^{2}}\right)\right]
$$




$$
\leq F_{\psi\left(\mathbb{C}^{2} \times U\right)}\left[(q(z))^{\gamma}\left(\alpha+\frac{\beta}{q(z)}+t \frac{z q^{\prime}(z)}{(q(z))^{2}}\right)\right] .
$$

The remaining part of Theorem 2.2 is similar to that of Theorem 2.1 and thus we omit it.

\section{References}

[1] K. O. Babalola, On $\lambda$-pseudo-starlike functions, J. Class. Anal. 3(2) (2013), 137-147. https://doi.org/10.7153/jca-03-12

[2] A. Haydar Eş, On fuzzy differential subordination, Math. Morav. 19(1) (2015), 123-129. https://doi.org/10.5937/MatMor1501123H

[3] A. Alb Lupaş, On special fuzzy differential subordinations using generalized Salagean operator and Ruscheweyh derivative, J. Adv. Appl. Comp. Math. 4 (2017), 26-34.

[4] A. Alb Lupaş, A note on special fuzzy differential subordinations using multiplier transformation and Ruschewehy derivative, J. Comp. Anal. Appl. 25(6) (2018), 11251131.

[5] G. I. Oros and Gh. Oros, The notion of subordination in fuzzy set theory, Gen. Math. 19(4) (2011), 97-103.

[6] G. I. Oros and Gh. Oros, Fuzzy differential subordination, Acta Univ. Apulensis 30 (2012), 55-64.

[7] G. I. Oros and Gh. Oros, Dominants and best dominants in fuzzy differential subordinations. Stud. Univ. Babeş-Bolyai Math. 57(2) (2012), 239-248.

[8] K. Sakaguchi, On a certain univalent mapping, J. Math. Soc. Japan 11(1) (1959), 72-75. https://doi.org/10.2969/jmsj/01110072

[9] L. A. Zadeh, Fuzzy sets, Information and Control 8 (1965), 338-353. https://doi.org/10.1016/S0019-9958(65)90241-X

[10] A. K. Wanas and A. H. Majeed, Fuzzy differential subordinations for prestarlike functions of complex order and some applications, Far East J. Math. Sci. (FJMS) 102(8) (2017), 1777-1788. https://doi.org/10.17654/MS102081777

[11] A. K. Wanas and A. H. Majeed, Fuzzy differential subordination properties of analytic functions involving generalized differential operator, Sci. Int. (Lahore) 30(2) (2018), 297302. 